\title{
Neuromuscular and Vascular Hamartoma: Starting Point in Crohn's Disease?
}

\author{
Samyak Dhruva, d, Shamsuddin Anwar ${ }^{\mathrm{a}}$, Meena Kashi ${ }^{\mathrm{b}}$, \\ Indraneil Mukherjee ${ }^{\mathrm{c}}$
}

\begin{abstract}
Neuromuscular and vascular hamartoma (NMVH) is a benign and rare lesion comprising abnormal proliferation of cellular elements in the mucosa of the small intestine. NMVH shares certain histological features with inflammatory conditions like Crohn's disease, radiation and ischemic enteritis, and has been a part of ongoing debate since it was first described in 1982. We intend to describe an interesting case of small bowel obstruction caused by NMVH and emphasize the importance of keeping it as an important differential diagnosis in cases of small bowel obstructions for general practitioners and gastroenterologists. After detailed analysis of the 28 cases of the NMVH in the literature we are also suggesting NMVH as a starting point in Crohn's disease, and if proven it will change the management strategies for Crohn's disease.
\end{abstract}

Keywords: Hamartoma; Neuromuscular and vascular hamartoma; Crohn's disease; Inflammatory bowel disease

\section{Introduction}

Neuromuscular and vascular hamartoma (NMVH) is an uncommon lesion of the small intestine which was first described by Fernando and McGovern in 1982 [1]. It is considered to be a benign proliferation of neural, vascular and muscular elements resulting in lesions like strictures or polypoid masses. These lesions can present in variable length along the small bowel wall. We are describing one such rare case of NMVH in distal ileum in a young female who presented with symptoms of small intestinal obstruction.

\section{Case Report}

A 44-year-old female with pertinent past surgical history of

Manuscript submitted May 26, 2021, accepted June 10, 2021

Published online July 3, 2021

a Department of Internal Medicine, Northwell Health, New York, NY, USA

${ }^{b}$ Department of Pathology, Northwell Health, New York, NY, USA

'Department of General Surgery, Northwell Health, New York, NY, USA

${ }^{\mathrm{d} C}$ Corresponding Author: Samyak Dhruv, Department of Internal Medicine,

Northwell Health, New York, NY, USA. Email: Samyakdhruv92@gmail.com

doi: https://doi.org/10.14740/jmc3727 cholecystectomy, currently on oral contraceptive medication only was evaluated for frequent and recurrent episodes of nausea and abdominal pain for the past few months. When the initial workup on the abdominal X-ray suggested small bowel obstruction, the patient was hospitalized and managed conservatively. The computed tomography (CT) of the abdomen and pelvis showed multiple dilated small bowel loops, interloop fluid level and transition point in the right lower quadrant (Fig. 1).

The patient continued to follow up at outpatient since the resolution of acute complaints. Upon further investigation, the magnetic resonance enterography (MRE) demonstrated resolution of small bowel obstruction with focal short segment fixed stricture in an ileal loop of the lower abdomen (Fig. 2). The endoscopy and colonoscopy did not yield any significant findings of inflammatory bowel disease or malignant disease pathology. Given the recurrent episodes of abdominal pain, the patient was offered diagnostic laparoscopy for further evaluation of the ileal stricture (Fig. 3). She underwent small bowel resection and anastomosis and eventual resolution of symptoms.

The ileal stricture showed ulceration with underlying thickened and disorganized muscularis mucosa and submuco-

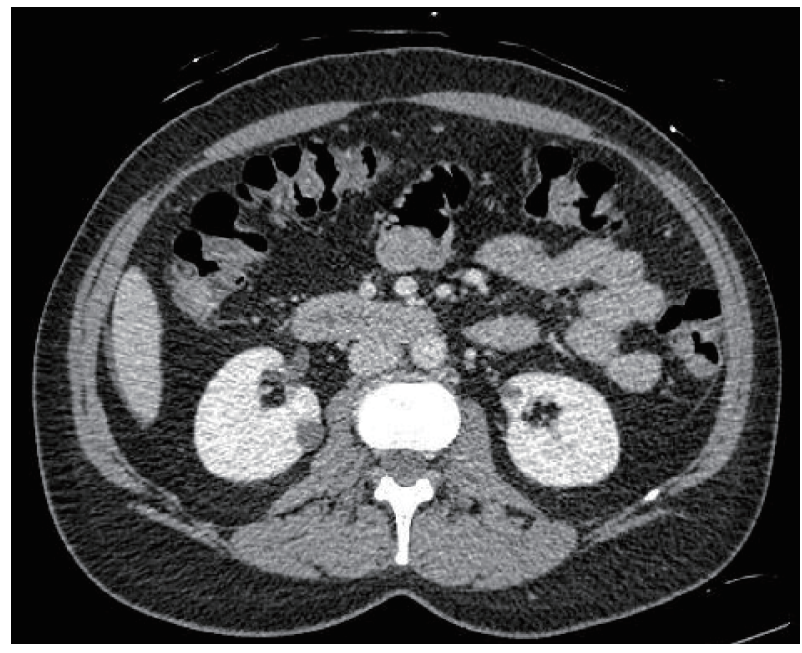

Figure 1. CT abdomen and pelvis with intravenous contrast showing multiple dilated small bowel loops with fecalization, interloop fluid and transition point in the right lower quadrant (colon not completely collapsed, and findings likely consistent with early small bowel obstruction). CT: computed tomography. 


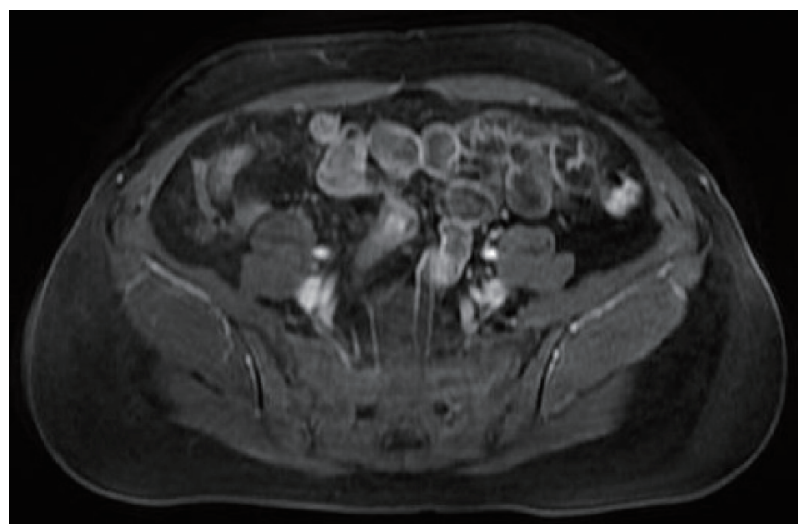

Figure 2. MRI enterography with and without intravenous contrast showing resolution of small bowel obstruction, focal, short segment luminal narrowing of an ileal loop in the lower mid abdomen in the region of previously seen transition point, and unremarkable appendix. MRI: Magnetic resonance imaging.

sa, increased number of ganglion cells, prominent vasculature and increased nerve bundles with no evidence of neoplastic, granulomatous inflammation or transmural lymphoid aggregates (Fig. 4). These findings were consistent with $\mathrm{NMVH}$ of the small bowel. The immunohistochemical analysis supported the diagnosis with synaptophysin and S-100 (Fig. 5) highlighting prominent ganglion cells (neural elements) and desmin stain (Fig. 5) showing disorganized bundles of smooth muscles.

As Crohn's disease was in the differential diagnosis for the segmental involvement of the small bowel wall with stricture, patient was also tested for biomarkers of inflammatory bowel diseases which resulted negative (Table 1).

\section{Discussion}

NMVH is defined as an abnormal, non-neoplastic proliferation of cellular elements normally found within the mucosa and submucosa of the gastrointestinal tract. Most common locations described in medical literature are jejunum and ileum and presenting in all age groups. Fernando and Mcgovern in 1982

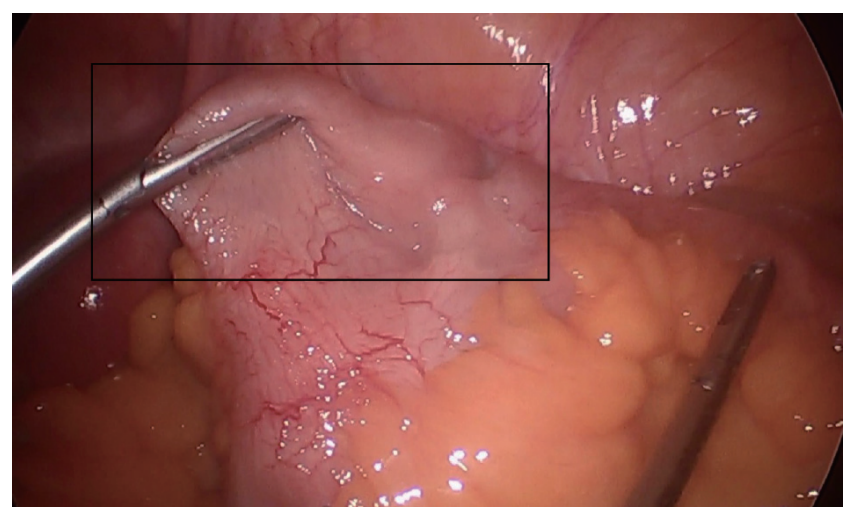

Figure 3. Ectatic small bowel on the laparoscopic image taken during the resection (black square showing entire ectatic segment).
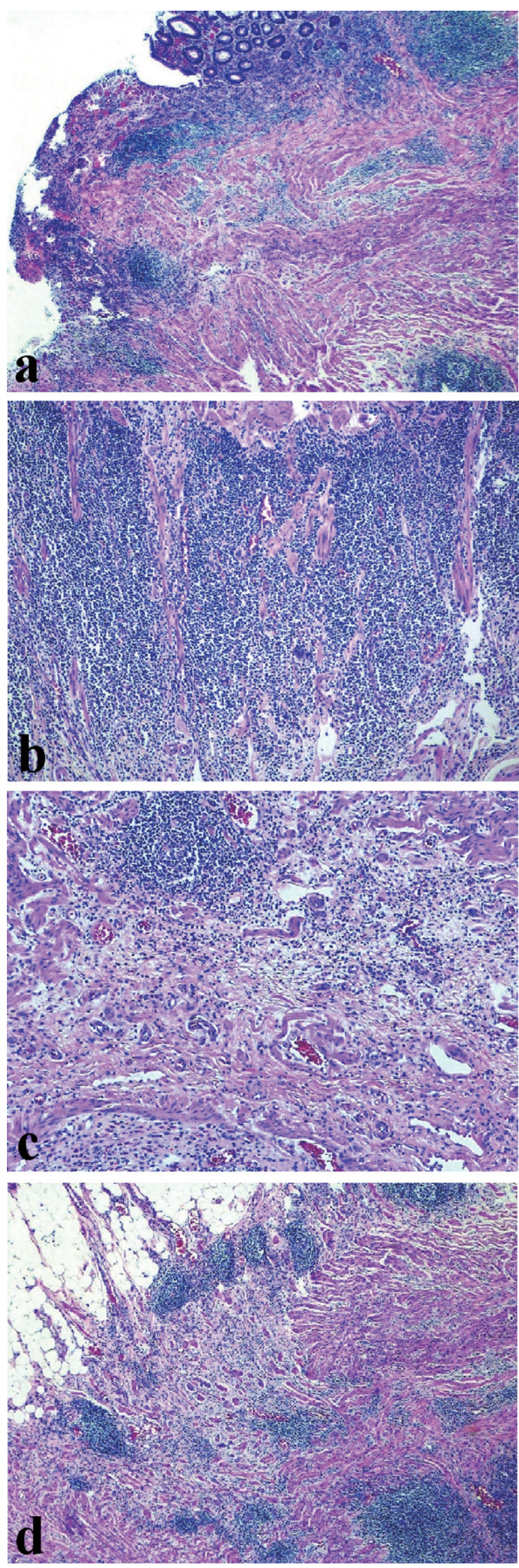

Figure 4. (a) Stricture area showing surface mucosal ulceration (H\&E, $\times 40$ ). (b) Dense mixed inflammatory infiltrate in the lamina propria $(H \& E, \times 100)$. (c, d) Thickened and disorganized muscularis mucosa and submucosa, prominent vasculature, prominent nerve bundles and clusters of ganglion cells. H\&E: hematoxylin and eosin stain. 


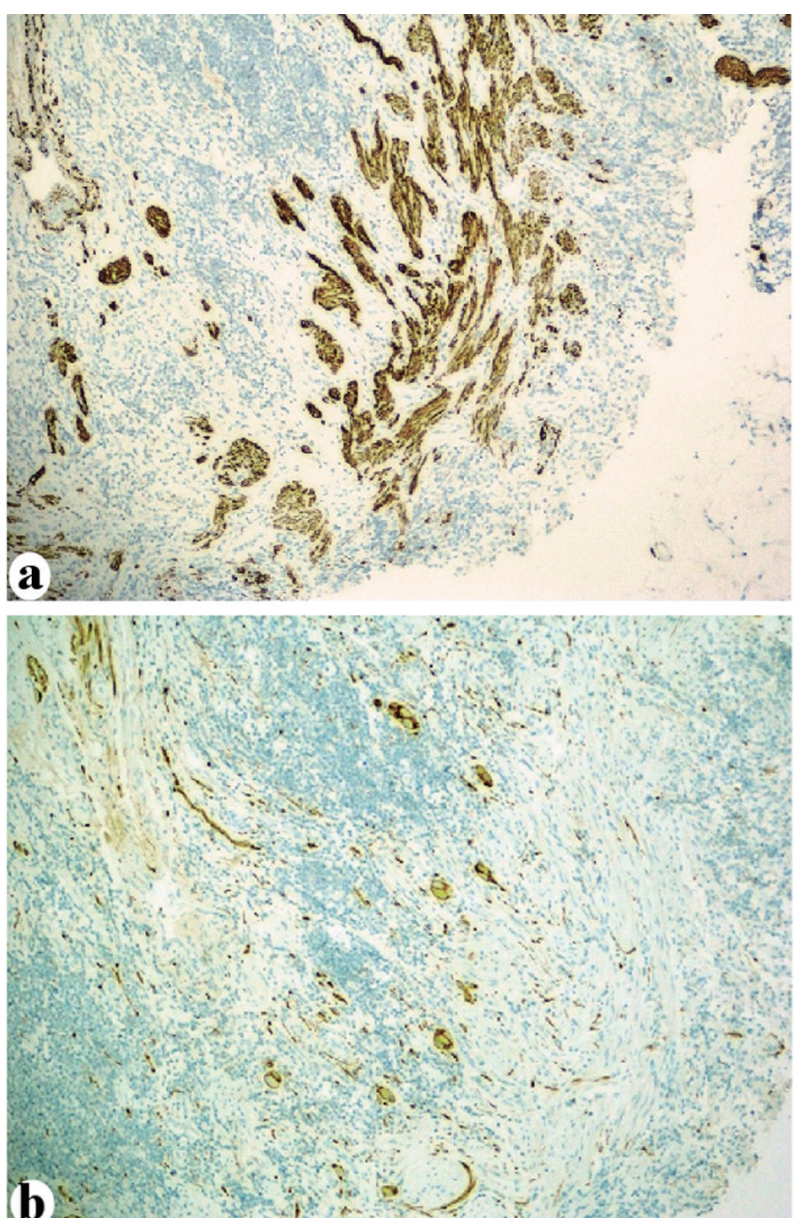

Figure 5. (a) Desmin immunostain highlights the disorganized bundle of smooth muscle. (b) S-100 highlights prominent ganglion cells and nerve fibers within the wall of intestine.

first coined the term "neuromuscular and vascular hamartoma" in their manuscript. It has been debated since then if the abovedescribed lesion represents as a separate entity or a "burnt-out" phase of Crohn's disease or secondary to radiation, ischemic enteritis [2].

To date, only 28 cases of NMVH have been reported including this one. The mean age of patient is 53 years, there are 10 males and 15 females with age unknown in four cases and sex is unknown in three cases. Out of these 28 cases, three had Crohn's disease as a comorbidity. NMVH originated in small intestine in 26 cases while appendix and cecum as the starting points in the other two cases Table 2 [1-21].

Histologically NMVH demonstrates disorganized elements of non-myelinated nerve fibers, ganglion cells, fascicles of smooth muscle, hemangiomatous vascular channels found in the mucosa and submucosa of the small bowel wall. A variety of immunohistochemical marker panel (smooth muscle actin, S100, vimentin, desmin, and cluster of differentiation (CD)3) and special stains (Masson trichrome, Verhoeff-vanGieson, and periodic acid-Schiff) can be utilized in identifying the components of NMVH as seen in our case report [22]. The most common presentation of such abnormal cell prolifera-
Table 1. Biomarkers for Inflammatory Bowel Disease (IBD)

\begin{tabular}{ll}
\hline Antibody & Result \\
\hline ASCA IgA & Negative \\
ASCA IgG & Negative \\
Anti-OmpC IgA & Negative \\
Anti-CBir 1 IgG & Negative \\
\hline Anti-FlaX IgG & Negative \\
\hline IBD-specific pANCA & Negative \\
ICAM-1 & Negative \\
VCAM-1 & Negative \\
VEGF & Negative \\
CRP & $13.2 \mathrm{mg} / \mathrm{L}$ \\
\hline
\end{tabular}

ASCA: anti-Saccharomyces cerevisiae antibody; Ig: immunoglobulin; Anti-Ompc: anti outer membrane porin C; Anti-CBir: anti-bacterial flagellin antibody; pANCA: perinuclear anti-neutrophil cytoplasmic antibody; ICAM: intracellular adhesion molecule; VCAM: vascular cell adhesion molecule; VEGF: vascular endothelial growth factor; CRP: C-reactive protein.

tions as seen in our patient are non-specific abdominal pain and obstructive features in the intestinal lumen. The other clinical manifestations described in medical literature include blood loss from the enteric tract or protein-losing enteropathy [5]. Given the scarcity of medical literature available for NMVH, the establishment of accurate diagnosis presents as a challenge for pathologists; however, the prognosis has been favorable after surgical resection and no documented recurrences [6].

NMVH has been thought to be a hamartoma consisting of an aberrant proliferation of neural, muscular, and vascular elements in the intestine [1]. However, Shepherd et al suggested that NMVH may represent an abnormal chronic histologic consequence of Crohn's disease [7]. However, considering that most of the patients of NMVH described in the literature did not exhibit prominent fibrosis of the intestinal wall when observed in the chronic inflammatory state, it is thought that NMVH may exist as a separate entity [8]. For such reasons, the debate continues as to whether this lesion is truly a hamartoma or represents a "burnt-out" phase of various chronic pathologies. Our case did not show any histological features such as prominent fibrosis that would indicate active or "burnt-out" Crohn's disease or another reactive process induced by chronic inflammation. The phase "burnt-out" of the Crohn's disease has long been used since Shephard et al [7] first used it, though it is very important to mention that his two out of four cases had already been diagnosed with active Crohn's disease; and the element of the NMVH lacked the classic Crohn's feature and so "burnt-out" word was used. Out of 28 cases described in the Table 2 [1-21], only three had Crohn's disease, and very close histological and anatomical similarities exist between the two. Because of that we suggest NMVH as a starting point of the Crohn's disease where the disease is not that advanced enough to show the transmural fissuring, if we can follow up the cases of the NMVH and look for the future development of the Crohn's disease, the development of NMVH will be estab- 
Table 2. Twenty-Eight Cases of NMVH so far in the Literature Describing the Authors, Year of Publication With Patient Characteristics and Comorbidities [1, 2, 3, 5-9]

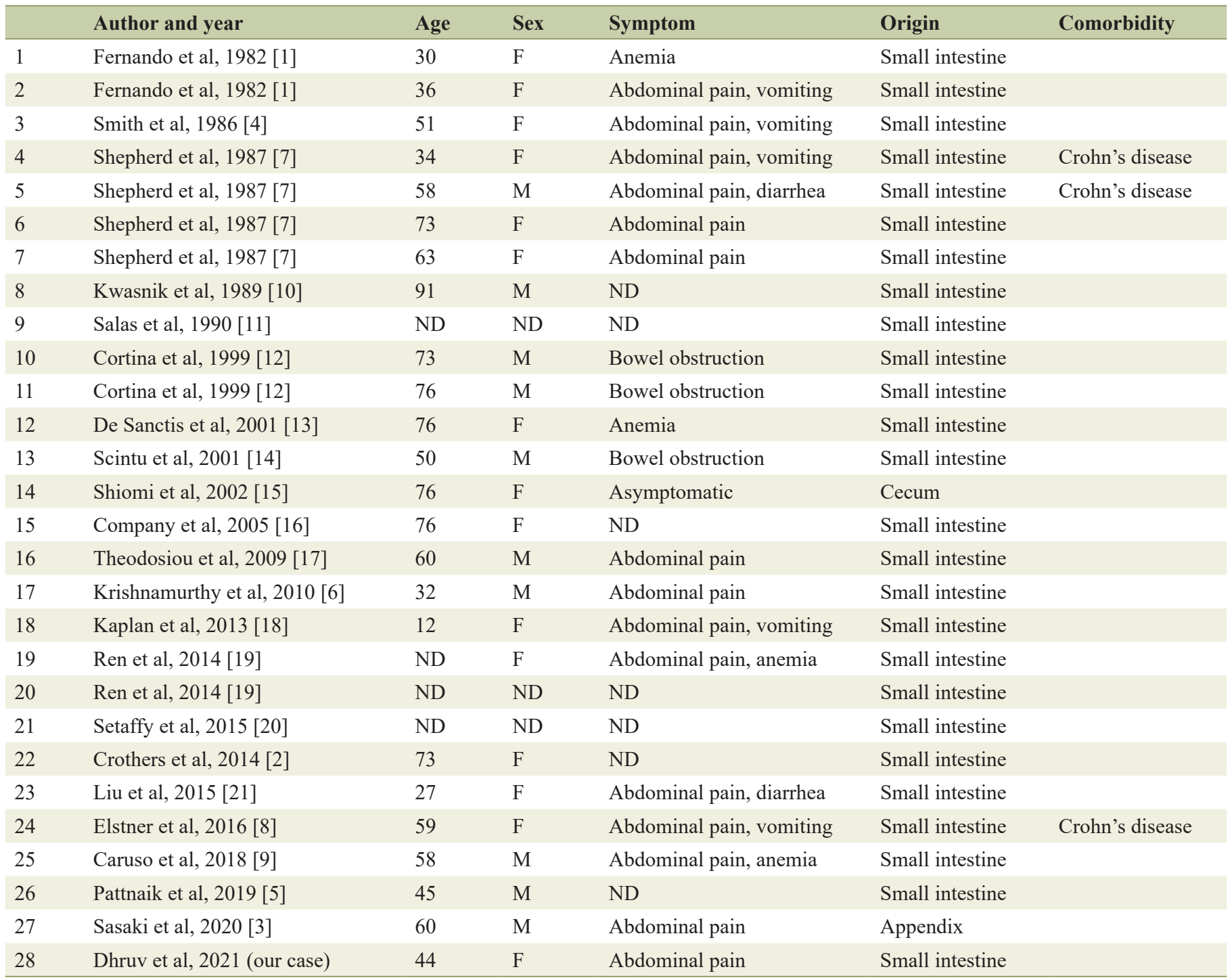

NMVH: neuromuscular and vascular hamartoma; F: female; M: male; ND: not detected.

lished as the "igniting event" in the development of the Crohn's disease, and that would have ground-breaking changes in the therapeutics for the Crohn's disease. Further, histopathological research is needed in this area and the link between two needs to be further microscopically scrutinized because there is a significant possibility of the connection, and any changes in the therapeutics will affect the lives of several people living with Crohn's disease around the world.

NMVH may mimic some clinical features associated with inflammatory bowel disease and other reactive conditions. The stricture seen in our patient initially raised the suspicion for Crohn's disease. However, there was no evidence of cobble stoning, fissures or fibrosis (evidence of Crohn's disease) on colonoscopy, and the exclusion of this diagnosis was also supported with biomarkers obtained. The absence of transmural chronic inflammation, granulomas and lymphoid aggregates upon microscopic analysis of the resected stricture established the diagnosis of NVMH in our patient. Other less common differentials like ischemic and radiation enteritis were not considered due to their irrelevance to our clinical case $[8,9]$.

\section{Learning points}

NMVH must be considered as important differential diagnosis in patients presenting with small intestinal stricture and obstructive symptoms. It is important to evaluate the patients in systematic way for common clinical entities like inflammatory bowel diseases, chronic inflammation and malignancy. Although very rare, NMVH is a benign abnormal proliferation of cellular elements with very good prognosis after curative resection. Because, out of 28 cases three cases had simultane- 
ous Crohn's disease, there is a possible link between the two. Though, previously described as the "burnt-out" phase of the Crohn's disease, not all cases of NMVH had Crohn's disease, so we suggest NMVH as a starting point in the Crohn's disease patients.

\section{Acknowledgments}

None to declare.

\section{Financial Disclosure}

All authors have declared that no financial support was received from any organization for the submitted work; and all authors have declared that there are no other relationships or activities that could appear to have influenced the submitted work.

\section{Conflict of Interest}

None to declare.

\section{Informed Consent}

Informed patient consent was obtained to write and publish this case report.

\section{Author Contributions}

Samyak Dhruv has written the manuscript. Shamsuddin Anwar and Meena Kashi helped with editing under the supervision of Indraneil Mukherjee. Samyak Dhruv is the submitting and corresponding author.

\section{Data Availability}

The authors declare that data supporting the findings of this study are available within the article.

\section{References}

1. Fernando SS, McGovern VJ. Neuromuscular and vascular hamartoma of small bowel. Gut. 1982;23(11):10081012.

2. Crothers JW, Zenali M. Neuromuscular and vascular hamartoma of the small intestine: an exuberant reparative process secondary to chronic inflammation. Int J Surg Pathol. 2015;23(8):673-676.

3. Sasaki T, Furuhata T, Nishimura M, Ono T, Noda A, Koizumi H, Miyajima N, et al. An extremely rare case of neuromuscular and vascular hamartoma of the appendix.
Surg Case Rep. 2020;6(1):216.

4. Smith CE, Filipe MI, Owen WJ. Neuromuscular and vascular hamartoma of small bowel presenting as inflammatory bowel disease. Gut. 1986;27(8):964-969.

5. Pattnaik SA, Mitra S, Mishra TS, Purkait S, Kumar P, Naik S. A vasculitis-associated neuromuscular and vascular hamartoma presenting as a fatal form of abdominal cocoon. Int J Surg Pathol. 2019;27(1):108-115.

6. Krishnamurthy V, Basavaraj V, Sunila, Vimalambike MG. Neuromuscular and vascular hamartoma of the small bowel: a rare cause of intestinal obstruction. J Lab Physicians. 2010;2(2):109-110.

7. Shepherd NA, Jass JR. Neuromuscular and vascular hamartoma of the small intestine: is it Crohn's disease? Gut. 1987;28(12):1663-1668.

8. Elstner KE, Vickers CR, Gett RM, Qiu MR, Killen LV. Neuromuscular and vascular hamartoma as an unusual cause of small bowel obstruction. Int J Surg Case Rep. 2016;26:1-3.

9. Caruso ML, Cavalcanti E, De Michele F, Ignazzi A, Carullo R, Mastronardi M. Small bowel capsule endoscopy revealing neuromuscular and vascular hamartoma of the jejunum: A case report. Medicine (Baltimore). 2018;97(15):e0196.

10. Kwasnik EM, Tahan SR, Lowell JA, Weinstein B. Neuromuscular and vascular hamartoma of the small bowel. Dig Dis Sci. 1989;34(1):108-110.

11. Salas A, Casellas F, Sanz J, Garcia F, Margarit C, Malagelada JR. Neuromesenchymal hamartoma of the small bowel. J Clin Gastroenterol. 1990;12(6):705-709.

12. Cortina G, Wren S, Armstrong B, Lewin K, Fajardo L. Clinical and pathologic overlap in nonsteroidal anti-inflammatory drug-related small bowel diaphragm disease and the neuromuscular and vascular hamartoma of the small bowel. Am J Surg Pathol. 1999;23(11):1414-1417.

13. de Sanctis S, Qureshi T, Stebbing JF. Clinical and pathological overlap in nonsteroidal anti-inflammatory drugrelated small bowel diaphragm disease and the neuromuscular and vascular hamartoma of the small bowel. Am J Surg Pathol. 2001;25(4):539-541.

14. Scintu F, Capra F, Giordano M, Frau G, Mascia R, Comella D, Rais M, et al. [Neuromuscular and vascular hamartoma of the small intestine. Report of a clinical case and review of the literature]. Chir Ital. 2001;53(3):393398.

15. Shiomi T, Kameyama K, Kawano Y, Shimizu Y, Takabayashi T, Okada Y. Neuromuscular and vascular hamartoma of the cecum. Virchows Arch. 2002;440(3):338340 .

16. Company MM, Usamentiaga E, Torralba J, Bonet L. [Small bowel diaphragm disease associated with NSAIDs with histology of neuromuscular and vascular hamartoma. Case report and literature review]. Gastroenterol Hepatol. 2005;28(4):228-231.

17. Theodosiou E, Voulalas G, Salveridis N, Pouggouras K, Manafis K, Christodoulidis K. Neuromesenchymal hamartoma of small bowel - an extremely rare entity: a case report. World J Surg Oncol. 2009;7:92.

18. Kaplan JL, Goldstein AM, Shenoy-Bhangle A, Badizade- 
gan K. Neuromuscular and vascular hamartoma of the small intestine in a child. J Pediatr Gastroenterol Nutr. 2013;56(5):e33-35.

19. Ren B, Cao W. Neuromuscular and vascular hamartoma: is it a true hamartoma? J Clin Pathol. 2014;67(3):284287.

20. Setaffy L, Osuna MJ, Plieschnegger W, del Pino Florez Rial M, Geboes K, Langner C. Cryptogenic multifocal ulcerous stenosing enteritis (CMUSE), and neuromuscular and vascular hamartoma (NMVH): two sides of the same coin? Endoscopy. 2015;47(4):345-348.

21. Liu N, Pan Y, Li ZS, Shi YP, Zhang XY, Wu GS, Wu $\mathrm{KC}$, et al. Neuromuscular and vascular hamartoma: a rare entity or special phase of Crohn's disease. J Dig Dis. 2015;16(1):52-54.

22. Ayyanar P, Purkait S, Mishra TS, Patra S, Mitra S. Histopathologic spectrum of neuromuscular and vascular hamartoma with special reference to the Vasculopathic 